\title{
A Model Of College Tuition Maximization
}

Donald I. Bosshardt, Canisius College, USA

Larry Lichtenstein, Canisius College, USA

Mark P. Zaporowski, Canisius College, USA

\begin{abstract}
This paper develops a series of models for optimal tuition pricing for private colleges and universities. The university is assumed to be a profit maximizing, price discriminating monopolist. The enrollment decision of student's is stochastic in nature. The university offers an effective tuition rate, comprised of stipulated tuition less financial aid, to each student based on the demographic characteristics of the student. Initially, the applicant poll is assumed to be homogeneous. Subsequently, the quality of the applicant pool is allowed to vary and the university's tuition maximization problem is subject to quality and capacity constraints. Lastly, we perform a simulation that allows an exploration of the risks associated with the university's tuition, quality and capacity decisions.
\end{abstract}

Keywords: Net Tuition Revenue Maximization, Uncertainty, Private Colleges

\section{INTRODUCTION}

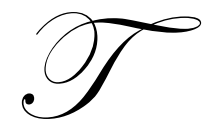

he primary focus of this paper is the explicit incorporation of environmental uncertainty into the economic modeling of the higher-education tuition decision. We argue that most of the existing literature explicitly ignores the implications of uncertainty at the theoretical level (for good reasons), and this brings many of the extant policy prescriptions into question. The immediate requirements for confronting uncertainty deal with the specification of the appropriate objective function for the college or university, and the issues associated with the formulation of constraints. Our solution is to suggest that in the absence of an appropriate composite utility function, a reasonable starting point is an expected profit maximizing strategy. This is not the end of the process, but rather it represents only the beginning stage of the analysis. We argue that further analysis (and simulation) is required to explore the uncertainty consequences of the decisions made.

The existing literature often ducks the issue of identifying the appropriate objective function under uncertainty. Even then, it exhibits considerable variability in the modeled objective function for the college/university. One common theme is some appeal to the economic theory of the firm. Unfortunately, the common appeal is to the theory of the firm under certainty and this ignores the necessary modifications associated with uncertainty. Jenny (1968) at first, likens the university to a firm, but then argues that the entity should be viewed as a 'multi-firm', a collection of firm-like units with economic optimization rules sometimes secondary to other objectives. In particular, Jenny points to the then prevalent balance-sheet divisions of current funds, student loan funds, endowment, other unexpendable and annuity funds, and plant funds, which he suggests are managed somewhat independently. He also recognizes the income components where a similar multi-firm phenomenon exists. While much of his discussion predates the decentralization literature in which the successful firm is required to develop decentralized objectives which are consistent with firm-wide optimization, Jenny goes on to argue that the result in practice is that:

trustees are trying to maximize some vague trusteeship function subject to all sorts of vague constraints ...

Jenny proceeds to model the firm in a 'satisficing' role where economic considerations are significant but cannot be quantified.

Ehrenberg and Sherman (1984) consider the problem of an elite institution in which tuition revenue is not an issue. They assume the university derives utility from 'quality units' of different categories, where the utility 
function has standard concavity properties ${ }^{2}$. They consider the objective function as unconstrained and their results are then formulated based upon elasticities.

Bryan and Whipple (1995) use a net revenue maximization model based on an assumed range of elasticities to first approximate alternative possible net tuition maximizing levels given their current student body, and then reestimate the tuition elasticity effects on net 'profit' using survey data over the indicated range of feasible choices. Their model ignores uncertainty, and neither does it consider quality or capacity issues. Perhaps because of these deficiencies, Bryan and Whipple go on to state:

However, the output of the TENEP (=their optimization) model is only one factor in the tuition decision process. Net earnings maximization may not be the major objective of the institution, as nonprofit goals may have higher priority. ${ }^{3}$

They conclude that their model can prove useful by establishing breakeven levels of tuition after the imposition of the other priorities.

Rothschild and White (1995) consider the social optimality of not explicitly pricing according to value added by the educational process. They argue that their formulation of the societal objective function leads to a characterization of

efficient prices which are also zero profit prices for the universities and thus are consistent with a competitive free entry equilibrium among the universities. ${ }^{4}$

While the Rothschild and White results are not directly applicable to this paper, they do go on to note that:

We assumed that universities were profit maximizers and (implicitly) that they were subject to the usual forms of resource constraints. But universities do not typically cover all their costs through tuition and other fees, and it is difficult to state what universities are maximizing or even who is doing the maximizing. ${ }^{5}$

They note that such ambiguities must be better understood before we will have a complete model of the social properties of the higher educational system. In a seminal paper dealing with competitive insurance markets, Rothschild and Stiglitz ${ }^{4}$ (1976), to rationalize the use of expected profit maximization for their competitive insurance firm, remarked:

Since the theory of the firm behavior under uncertainty is one of the more unsettled areas of economic theory, we cannot look to it for support of any assumption we might make... 6 .

We will pick up on this point towards the end of this section.

Martin (2004) considers the question of tuition discounting to an institution that wishes to maximize 'prestige' by maximizing alumnus success in the long run. He argues (and presents a formal model in a related paper Martin (2003)) that if 'prestige' is the long run goal and if the institution is subject to economies of scale or scope, then:

If the institution maximizes the human capital acquired by each student enrolled in the short run, then enrolling the highest quality student body consistent with the institution's quality reputation and its financial resources can maximize alumni success. Hence, in the short run the institution maximizes the quality of each class enrolled. ${ }^{7}$

Martin argues that his model demonstrates the incentive compatibility of short run quality maximization (subject to a balanced budget) with long run prestige maximization. His assumed objective is a prestige-utility function, which is related to alumni successes.

The parallels to the theory of the firm in this literature miss many of the important issues associated with uncertainty. In the theory of the firm under uncertainty, profit maximization is replaced by value maximization (an 
inter-temporal extension) which can be shown to be Pareto optimal assuming that markets are perfect and complete, and firms behave competitively. Value maximization is related to the observation that the objective function of the firm should be to maximize the utility of its owners. In general there is no way to form a composite utility function representing the owners, and only under conditions of perfect competition (and perfect capital markets) does wealth maximization guarantee unanimity among owners ${ }^{8}$.

A model of utility maximization may be appropriate for institutions that have such a deep applicant pool that generating revenues sufficient to cover the costs of operation are not in question. We believe that most institutions are not in such a position and must frequently cut costs if they do not bring in sufficient revenues. Hence the appropriate objective is some form of tuition maximization (or by duality cost minimization).

The paper proceeds as follows: the next section details a sequence of models represent our first pass at modeling the institution's tuition choices. Section 3 presents a simulation related to a simple version of our model. Section 4 concludes with some observations drawn from the models presented.

\section{THE MODEL}

This section details a sequence of economic models of expected profit (total tuition net of expenses) maximization for colleges and universities. We begin with the unconstrained problem -- the college wants to set tuition such that total 'profit' is maximized. We accept the observation that many/most Colleges and Universities display some level of risk aversion in their decision process, but we argue that except for those schools with significant endowments, these decisions may be made at the institution's peril. It would be nice to have a utility function to maximize, but given the horizon of successful universities, this would seem to be fool's errand. In the absence of such a utility function, we proceed with our simple model, assuming that it represents a close approximation to maximizing the probability of survival. In most respects, our analysis resembles the economic models of monopoly and monopolistic competition. We then move on to the issues of quality and capacity constraints. Our main results are summarized at the end of the paper.

\subsection{The Objective Function}

It is well known in Economics and Finance that the incorporation of uncertainty imposes more stringent requirements on the specification of the objective function ${ }^{9}$. For instance, the consumption decision is unique within monotonic transformations of the utility function under conditions of certainty. Hence, we only need to characterize preferences within a somewhat broad class of functions to arrive at the appropriate optimization. Under uncertainty, the uniqueness is limited to linear transformations -- a much more restrictive requirement. Thus we must be more precise in the specification of the objective function (assuming its non-linear) when uncertainty is a part of the problem. This paper develops a model of optimal tuition policy assuming that the institution is a profit maximizer and the student's enrollment decision is probabilistic in nature. Although many in academia may be loathe to treat the university as a profit maximizing firm, the institution's decision makers (president and board of trustees) may have good reason to do so. These include:

- $\quad$ In the current academic environment, failure to adopt profit maximizing pricing may threaten the financial stability of the institution. The institution may be forced to engage in profit maximizing behavior to survive.

- $\quad$ Profits generated by the institution can be used to increase the endowment fund, which can provide a financial cushion against future uncertainties, support new infrastructure and provide funding for scholarships based on pure need.

The second important feature of our model is the explicit incorporation of uncertainty. We believe that the number of institutions that are certain in July about the number of fall matriculants are few indeed. Modern decision theory tells us that decisions (at least in part) shape the probability distributions which govern future outcomes, and models which do not endogenize these effects are at best incomplete. As a practical matter, it would come as no surprise to faculty members that because of contracts, choices to remain with a college, and transactions costs 
associated with departing, many of the risks created by the tuition/matriculation decisions of colleges and universities are transferred in substantial part to the faculty.

\subsection{The Single Quality Class Model}

To develop the simplest aspects of the model we concentrate on a pool of homogeneous student applicants of quality. The non-quality characteristics affect the predisposition of the student to attend the college because of considerations other than quality (i.e.I family alumni status, locality, etc.). The depth of the applicant pool is $N$ and is assumed exogenous. The college makes an offer to the applicant in the form of a net tuition $T{ }^{10}$ The applicant accepts this offer with probability $p$, which is assumed to be a decreasing function of net tuition cost, a decreasing function of student quality, and an increasing function of the non-quality, predisposition characteristic(s):

$$
p=p(T, \alpha, \beta), \quad \frac{\partial p}{\partial T}<0, \quad \frac{\partial p}{\partial \alpha}<0, \quad \frac{\partial p}{\partial \beta}>0 .
$$

The expected profit for the institution associated with this pool of students is $N p(T-v)-F$, where $v$ is the variable cost associated with the student if they accept, $F$ are the fixed costs of the institution, and $N$ is the depth of the pool (assumed exogenous at this point). The school chooses the net tuition offer to maximize expected profit. We assume that profit maximization occurs as an interior solution $(0<T<\infty)$. In that case, the first order condition characterizing the solution is:

$$
N \frac{\partial p}{\partial T}(T-v)+N p=0
$$

which is independent of the depth of the student pool $(N)$. This equation indicates that tuition should be set at the level such that the expected profit associated with the additional dollar of tuition ( $\mathrm{p} \times$ \$1) just offsets the expected net profit loss associated with the decrease in probability of receiving the net tuition $[-(T-v)(\partial \mathrm{p} / \partial \mathrm{T})]$. Another interpretation of the first order condition is that the probability elasticity of tuition should be set equal to minus one at the optimum. This follows from a simple manipulation of (1):

$$
N \frac{\partial p}{\partial T}(T-v)+N p=0 \Rightarrow \frac{1}{p} \frac{\partial p}{\partial T}(T-v)+1=0 \Rightarrow \varepsilon_{p}=-1
$$

where $\varepsilon_{p}$ is the elasticity of the probability of matriculation with respect to net tuition ${ }^{11}$. We can rule out the possibility that $p$ is a globally increasing function of $T$ because $p$ is necessarily non-negative, and if the probability of attracting a student were an increasing function of net tuition, the optimal policy would be to set the tuition at infinity.

According to equation (1), the level of variable costs tends to attenuate the level of net tuition. For a given probability of enrollment and a given response to net tuition changes $(\partial p / \partial T)$, the higher the level of variable costs, the higher will be the net tuition.

\subsection{A The Second Order Condition}

If the solution to the first order condition is to represent a(n interior) maximum, the second order condition requires that:

$$
\frac{\partial^{2} p}{\partial T^{2}}(T-v)+2 \frac{\partial p}{\partial T}<0
$$


We have already assumed that $p$ is decreasing in net tuition so the last term on the left hand side of (2) is negative. A sufficient condition for a maximum exists if the second derivative of the probability with respect to net tuition is either zero or negative (and the deal is profitable to the school). However, if probabilities are to remain a non-negative function of tuition, then the second derivative must be positive. A minimum may then not exist. An example would be where the probability of matriculation diminishes to .5 for infinite tuition levels. The optimal policy would be to charge an infinite tuition level and expected profits would be infinite. From this example it seems clear that we must enforce the restriction (along with $p(T) \geq 0$ ) that $p(\infty)=0$ ), and the sufficient condition would then be $\quad 0 \leq \frac{\partial^{2} p}{\partial T^{2}}(T-v)<2 \frac{\partial p}{\partial T}$.

This equation simply states that it is necessary that the second derivative $\left(\partial^{2} p / \partial T^{2}\right)$ is of small magnitude when compared to $(\partial p / \partial T)$.

\subsection{B Comparative Statics}

One question often posed by economists is how the appropriate instrument (net tuition) changes as the parameters (here the quality variable) change. Because the optimal instrument is defined through the appropriate first order conditions, it is mathematically accurate to perturb the parameter and then adjust the instrument so that first order condition(s) is again satisfied. A slight change $(\mathrm{d} \alpha)$ in the parameter $\alpha$ would change the value of the first derivative (1) to:

$$
N\left(\frac{\partial^{2} p}{\partial T \partial \alpha}(T-v)-\frac{\partial p}{\partial T} \frac{\partial v}{\partial \alpha}+\frac{\partial p}{\partial \alpha}\right) d \alpha
$$

In response, the institution would reset tuition. By the assumed continuity of the partial derivatives, the offset is:

$$
\left(\frac{\partial^{2} p}{\partial T^{2}}(T-v)+2 \frac{\partial p}{\partial T}\right) d T
$$

This change would restore the first derivative to zero. The final equation becomes:

$$
\left(\frac{\partial^{2} p}{\partial T \partial \alpha}(T-v)-\frac{\partial p}{\partial T} \frac{\partial v}{\partial \alpha}+\frac{\partial p}{\partial \alpha}\right) d \alpha+\left(\frac{\partial^{2} p}{\partial T^{2}}(T-v)+2 \frac{\partial p}{\partial T}\right) d T=0
$$

Solving for the appropriate change in $T$ in response to a quality change yields:

$$
\frac{\partial T}{\partial \alpha}=-\frac{\partial^{2} p / \partial T \partial \alpha(T-v)-\partial p / \partial T \partial / \partial \alpha+\partial p / \partial \alpha}{\partial^{2} p / \partial T^{2}(T-v)+2 \partial p / \partial T}
$$

The right hand side denominator is our second order condition and is therefore negative. Therefore, the sign of the response is the sign of the numerator. In the numerator, the last term is negative. If variable costs are not increasing in quality, the middle term is also negative or zero, and the first term depends upon how $\partial p / \partial T$ changes as $\alpha$ is increased. We sign this response as negative indicating that the school would find it optimal to charge lower tuition to higher quality students. 


\subsection{Many Quality Classes}

Suppose that there are $k$ quality classes and these are ordered with $\alpha_{1}<\alpha_{2}<\ldots<\alpha_{k}$. In each quality class there are $N_{\mathrm{i}}$ potential students. We denote the net tuition for each class as $T_{\mathrm{i}}{ }^{12}$ In this setting, expected tuition profit is:

$$
\text { ExpectedProfit }=\sum_{i=1}^{k}\left[p_{i} N_{i}\left(T_{i}-v_{i}\right)-F_{i}\right]
$$

We rewrite the probability of matriculation as a function of net tuition and assume the following properties:

$$
p_{i}=p_{i}\left(T_{i}, \alpha_{i}, \beta_{i}\right), \quad \frac{\partial p_{i}}{\partial T_{i}}<0, \quad \frac{\partial p_{i}}{\partial \alpha_{i}}<0, \quad \frac{\partial p_{i}}{\partial \beta_{i}}>0
$$

Note that the characterization (5) explicitly assumes that the probability of a particular quality class is independent of tuition charged to other classes. The first order conditions characterizing expected profit maximization are:

$$
N_{i} \frac{\partial p_{i}}{\partial T_{i}}\left(T_{i}-v_{i}\right)+N_{i} p_{i}=0 \quad \text { for } 1 \leq i \leq k
$$

This is a set of first order conditions which must hold simultaneously for each of the $k$ quality classes. Note that the depth of each pool does not affect the solution, and the optimal level of net tuition for each quality pool satisfies the pool equivalent of (1).

\subsection{A The Quality Constraint}

The random nature of realized quality makes the specification of the quality constraint problematic. Realized quality is a fractional ratio based on random variables. Were we to require that an expected quality level be met, or met with some probability, the exact nature of the distribution would be required. We could argue that realized quality would be approximately normal based upon our simulation results reported in the next section and proceed in that vein using estimates for expected enrollment and standard deviation which embody the tuition choice consequences. We find it more convenient to follow the somewhat simplistic, but slightly more consistent approach of requiring that the total expected quality points meet a certain level relative to the expected enrollment. This undoes the ratio of random variables issue which obscured the explicit nature of the probability distribution. We express the constraint as:

$$
\Sigma N_{i} p_{i} \alpha_{i}-N^{*} \bar{\alpha} \geq 0
$$

where the summed component represents the total quality of the enrolled class, the quantity $\mathrm{N}^{*}$ is the expected number of enrolled students,

$$
N^{*}=\sum_{i=1}^{k} N_{i} p_{i}
$$

and the second term on the left hand-side of (7) is the target total quality points for the enrolled number of students.

We write the objective function for the tuition problem as: 


$$
\operatorname{Max} \sum_{i=1}^{k}\left[p_{i} N_{i}\left(T_{i}-v_{i}\right)-F_{i}\right]+\lambda\left(\sum_{i=1}^{k} N_{i} p_{i} \alpha_{i}-N * \bar{\alpha}\right) .
$$

The last parenthesis is associated with the quality constraint, (7), and the multiplier $\lambda$ is the shadow cost of the (per student enrolled) quality constraint ${ }^{13}$. The Kuhn-Tucker conditions associated with the optimization are:

$$
\begin{aligned}
& N_{i} \frac{\partial p_{i}}{\partial T_{i}}\left(T_{i}-v_{i}\right)+N_{i} p_{i}+\lambda N_{i} \frac{\partial p_{i}}{\partial T_{i}}\left(\alpha_{i}-\bar{\alpha}\right)=0 \\
& \Sigma N_{i} p_{i} \alpha_{i}-N^{*} \bar{\alpha} \geq 0 \\
& \lambda\left(\Sigma N_{i} p_{i} \alpha_{i}-N^{*} \bar{\alpha}\right)=0 .
\end{aligned}
$$

Equation (10) is the average quality constraint. Equation (11) is a Kuhn-Tucker equation which indicates that when the constraint is not binding (i.e. if $\Sigma N_{i} p_{i} \alpha_{i}-N^{*} \bar{\alpha}>0$ ), the shadow price of the constraint is to be interpreted as zero. This might be the case for an Ivy-League school which has adequate pool depths at even the highest quality levels. In that case, equation (9) reduces to the set of equations represented by (6) because the problem is an effectively unconstrained optimization.

When the quality constraint is binding, Equation (9) indicates that net tuition will be set at lower levels than in the unconstrained case for students whose quality level is above the desired average, and will be set above the unconditional optimal when the quality level is below the desired average. For a student whose quality level is exactly at the desired quality, the shadow price term is zero. Because we assume no interaction effects, the first order condition for those students will be exactly the same as in the unconstrained case, and the resulting tuition will be exactly the same. This follows from the observation that the quantity $\lambda$ is positive by construction, the probability derivative is negative, and thus the sign of the final term depends upon the difference between student quality and the desired average ${ }^{14}$. The last term is positive for low quality students, indicating that their tuition would be above the unconstrained optimum, and is below for high quality students indicating that a discount to their tuition be offered to encourage matriculation.

\subsection{B Quantity/Capacity Constraints}

We now suppose the college faces a capacity constraint. As noted in Martin (2002), there are at least three types of capacity in the modern College. They are physical plant capacity, instructional capacity, and subsidy capacity (endowment cash flows to the operating divisions). There clearly is a long run issue associated with underenrollment given the capacities of the institution, but these might be thought to primarily threaten the college's long run survival. This particular formulation embodies an expected capacity limit for the institution. Let $C$ be the maximum capacity. The objective function is to maximize the following expression which represents expected profit subject to the capacity constraint:

$$
\sum_{i=1}^{k}\left[p_{i} N_{i}\left(T_{i}-v_{i}\right)-F_{i}\right]+\delta\left(C-\sum_{i=1}^{k} N_{i} p_{i}\right),
$$

where $\delta$ is the shadow price associated with the capacity constraint. The associated first order conditions are:

$$
N_{i} \frac{\partial p_{i}}{\partial T_{i}}\left(T_{i}-v_{i}\right)+N_{i} p_{i}-\delta N_{i} \frac{\partial p_{i}}{\partial T_{i}}=0
$$




$$
\begin{aligned}
& C-\sum_{i=1}^{k} N_{i} p_{i} \geq 0 \\
& \delta\left(C-\sum_{i=1}^{k} N_{i} p_{i}\right)=0 .
\end{aligned}
$$

As before, equation (13) is the capacity constraint. Equation (14) is a Kuhn-Tucker equation which indicates that when the constraint is not binding, the shadow price of the constraint is to be interpreted as zero. Equation (12) is the first order condition associated with expected tuition profit maximization. Note that a binding capacity constraint raises the net tuition to all groups but more so for more tuition sensitive pools. In that case, equation (12) reduces to the set of equations represented by (6) because the problem is an unconstrained optimization.

\subsection{Summary}

In this section we have investigated a simple model of expected (net) tuition maximization for colleges and universities. The model avoids the objective function issues discussed in the introduction. In the next section we will employ the model in a simplified setting to focus on the risk and return trade-offs which are the consequences of this behavior for the institution.

\section{A LINEAR MODEL AND ITS SIMULATION}

The following section describes a simulation associated with a simplified version of the model previously developed. The model is characterized by linear probabilities of matriculation. We do so with the explicit hope that these simple functions would prove estimable. The primary focus is on the variability of outcomes.

\subsection{A Single Quality Pool}

We begin the analysis with a single quality pool. The college makes an offer to each of the $N$ applicants in the form of a net tuition offer $T$. This offer does not vary among applicants. The applicant accepts this offer with probability $p$, which is assumed to be a linear function of net tuition but not dependant on the identity of the individual:

$$
p=a-b T, \quad 0<a \leq 1, \quad b>0, \quad T<a / b,
$$

where $a$ and $b$ are assumed to be functions of the parameters $\alpha$ and $\beta$. The acceptance or rejection of the offer by any pool member is assumed to be independent of the decision by any other pool applicant. The actual profit, a random variable to the institution, is determined by the number of actual matriculants, $n$, and the net profit per student. Let $\pi$ denote this profit level, then $\pi=n(T-v)-F$,

where $v$ is the variable cost associated with the student if they accept and $F$ are the fixed costs to the institution (independent of the number of enrollees) associated with that group. Because $n$ is the (only) source of randomness, and because $\mathrm{n}$ is binomially distributed under our assumptions, the expected profit for the institution is:

$$
\mathrm{E}(\pi)=N p(T-v)-F .
$$

The school chooses the net tuition offer to maximize expected profit (16). We assume that expected profit maximization occurs at a $T^{*}$ where the probability of matriculating is nonnegative. In that case, the first order condition characterizing the optimal net tuition is:

$$
N(-b)(T-v)+N(a-b T)=0,
$$


which is independent of the depth of the student pool $(N)$. It is easily verified that the solution to the first order condition is:

$$
T^{*}=\frac{a}{2 b}+\frac{v}{2}
$$

Equation (18) indicates that the optimal tuition increases with the propensity to attend (a), and decreases with the tuition sensitivity of the applicant pool $(b)$. Equation (18) also indicates that the level of variable costs increases the net tuition by one-half its magnitude. Substituting (18) back into (15) yields the following probability of matriculation associated with the optimal tuition:

$$
p^{*}=(a-b v) / 2 .
$$

This equation indicates that the real constraint on our parameter choices is $(a-b v \geq 0)$. Any other choice of parameters implies a negative probability of matriculation and invalidates (16) which assumes that $\mathrm{Np} \geq 0$. Equation (19) is also prescriptive in that it indicates that a college should never court a group of students whose propensity to attend is less than the tuition sensitivity times the variable cost level.

To illustrate our model, we suppose that the college is dealing with an applicant pool with the following characteristics: $\mathbf{a}=1.00, \mathbf{b}=.0001, \mathbf{N}=5000$ and $\boldsymbol{\alpha}=.25$. For simplicity, we assume there are no variable costs. From (18), the optimal solution is to charge tuition of $\$ 5000$ resulting in an expected enrollment of 2500 students, a probability of enrollment of .5 , and expected revenue of $\$ 12.5 \mathrm{MM}$. The actual number of students would follow a binomial distribution with the stated mean and a variance of 1250. This implies a standard deviation of roughly 35.36 students. Using the normal approximation, enrollment should fall in the 2431 to 2569 range approximately 95 percent of the time. Equivalently, the 95 percent confidence interval for realized 'profit' would have a range of $\$ 12.155 \mathrm{MM}$ to $\$ 12.845 \mathrm{MM}^{15}$.

\subsection{Two Quality Classes}

To make the specification more interesting, suppose that there are two quality classes which we shall label high quality and low quality. The low quality pool has $\mathrm{N}_{\mathrm{L}}$ members, while the high quality pool has $\mathrm{N}_{\mathrm{H}}$ members. In this setting, expected net tuition is:

$$
\mathrm{E}(\pi)=N_{L} p_{L}\left(T_{L}-v_{L}\right)-F_{L}+N_{H} p_{H}\left(T_{H}-v_{H}\right)-F_{H} .
$$

We continue with the linear probability model and let

$$
\begin{aligned}
& p_{L}=a_{L}-b_{L} T_{L}, \\
& p_{H}=a_{H}-b_{H} T_{H} .
\end{aligned}
$$

Note that this specification denies any interaction of tuition (or matriculants) from the other quality pool (i.e. the tuition charged to the low quality pool does not affect the probability of matriculation for the high quality entrants).

\subsection{A The Unconstrained Solution}

If the college simply wishes to maximize expected net tuition, the first order conditions characterizing the optimal tuition levels are independently determined as:

$$
\begin{aligned}
& N_{L}\left(-b_{L}\right)\left(T_{L}-v_{L}\right)+N_{L}\left(a_{L}-b_{L} T_{L}\right)=0 \\
& N_{H}\left(-b_{H}\right)\left(T_{H}-v_{H}\right)+N_{H}\left(a_{H}-b_{H} T_{H}\right)=0 .
\end{aligned}
$$


The depth of the pool does not affect the solution, and the optimal level of net tuition for each quality pool satisfies the pool equivalent of (18). by:

For concreteness, we will assume that the parameters characterizing our two quality populations are given

\begin{tabular}{ccccc} 
Group & $\mathbf{a}$ & $\mathbf{b}$ & $\mathbf{N}$ & $\boldsymbol{\alpha}$ \\
\hline $\mathrm{L}$ & 1.00 & .0001 & 5000 & .25 \\
$\mathrm{H}$ & .60 & .0001 & 2000 & .75
\end{tabular}

Fixed and variable costs are taken to be zero $\left(v_{i}=F_{i}=0\right)$. The first order conditions can be solved directly in this example and lead to the unconstrained solution to charge tuition of $\$ 5000$ to Low Quality students (just as before) and $\$ 3000$ to High Quality students. The probabilities of matriculation are .5 for the low quality group and .3 for the high quality group. This leads to expected matriculations of 2500 low quality students and 600 high quality students. Both probability elasticities can be verified to be -1 at the optimal solution. This tuition policy results in expected total profit of \$14.3MM and an expected enrollment of 3100 students. The student body quality level at this expected enrollment is .3468 .

As previously indicated, actual tuition (which is the sum of two binomial variates) is a random variable and its probability density can be approximated through simulation. The actual distribution is a mixture of drawings from two separate populations. Figure 3 portrays a histogram of 10,000 realizations of the tuition outcome and the associated normal approximation. Note the adequacy of the normal approximation. The average tuition revenue was $\$ 14.298 \mathrm{MM}$ while the sample standard deviation was $\$ 189,780^{16}$. Figure 1 indicates that actual tuition varies between $\$ 13.6$ and $\$ 15.0$ million.

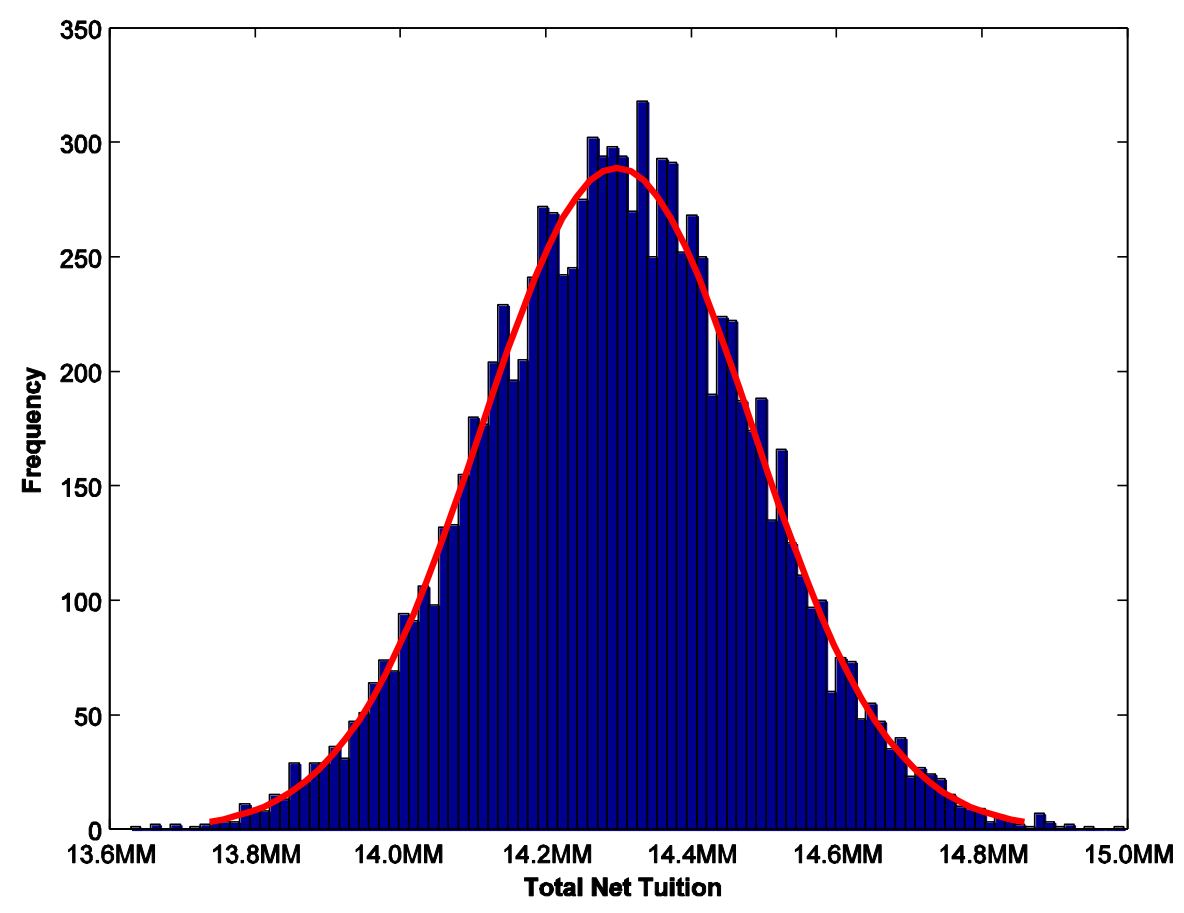

Figure 1 
The smooth curve visible in this figure is the density for a normal distribution with the mean and standard deviation displayed in the sample. It is noteworthy that the standard deviation for tuition revenue has only increased slightly from its single population value of $\$ 176,800$.

Perhaps of more interest is the associated distribution of student quality. Realized quality is defined as:

$$
\text { Quality }=\frac{.25 n_{L}+.75 n_{H}}{n_{L}+n_{H}} \text {, }
$$

and is hence a random variable. The exact distribution is unclear to us, but Figure 2 portrays the quality realizations for the 10,000 random samples which generated the tuition distribution in Figure 1. This suggests that the normal distribution is a reasonable approximation to the quality distribution.

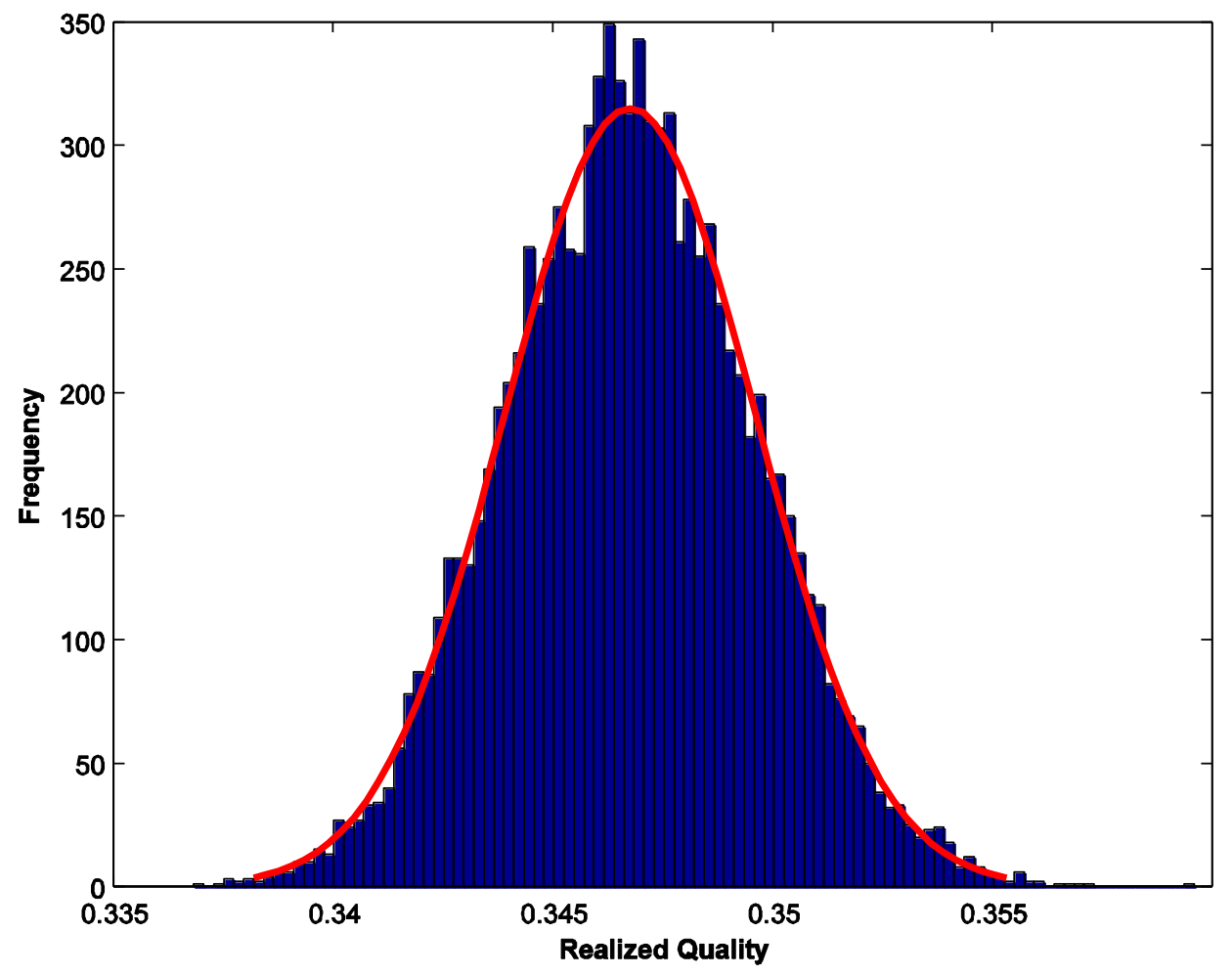

Figure 2

The sample mean and standard deviation are .3468 and .0029 . As before, the smooth curve is the normal probability density with the appropriate mean and standard deviation. The sample mean agrees well with the quality level of the expected enrollment ${ }^{17}$.

\subsection{B A Desired Quality Constraint}

In Section 2.3.A, we discussed the general issues associated with the specification of the quality constraint. In the next simulation we follow the simple path discussed there. We assume the school wishes to set a per expected number of matriculants quality level at $\bar{\alpha}$. The constraint is expressed as:

$$
N_{L} p_{L} \alpha_{L}+N_{H} p_{H} \alpha_{H}-N^{*} \bar{\alpha} \geq 0
$$


where the summed component represents the total expected quality points based upon expected enrollment. The quantity $N^{*}$ is the expected number of enrolled students,

$$
N^{*}=N_{L} p_{L}+N_{H} p_{H}
$$

and the term $N^{*} \bar{\alpha}$ is the target total quality points for the number of students expected to enroll. The objective function for the institution becomes:

$$
\operatorname{Max} \sum_{i=1}^{k}\left[p_{i} N_{i}\left(T_{i}-v_{i}\right)-F_{i}\right]+\lambda\left(\sum_{i=1}^{k} N_{i} p_{i} \alpha_{i}-N^{*} \bar{\alpha}\right)
$$

The last parenthesis is associated with the quality constraint, $\left(\Sigma N_{i} p_{i} \alpha_{i}-N^{*} \bar{\alpha} \geq 0\right.$, and the multiplier $\lambda$ is the shadow cost of the (per student enrolled) quality constraint ${ }^{18}$. The first order conditions associated with the optimization are:

$$
\begin{aligned}
& N_{i} \frac{\partial p_{i}}{\partial T_{i}}\left(T_{i}-v_{i}\right)+N_{i} p_{i}+\lambda N_{i} \frac{\partial p_{i}}{\partial T_{i}}\left(\alpha_{i}-\bar{\alpha}\right)=0 \\
& \lambda\left(\Sigma N_{i} p_{i} \alpha_{i}-N^{*} \bar{\alpha}\right)=0 .
\end{aligned}
$$

Continuing our example, suppose the school wishes to achieve a quality level of .5. Using any available numerical optimization package (we used Solver in EXCEL and MatLab), the constrained solution is to charge a tuition of $\$ 7714$ to low quality students and $\$ 286$ to high quality students. This results in a total expected profit of $\$ 9.1431 \mathrm{MM}$, an expected enrollment of 2286 students, and the desired average quality of $.5^{19}$. Figure 3 depicts the simulation results for net tuition receipts as a result of this policy.

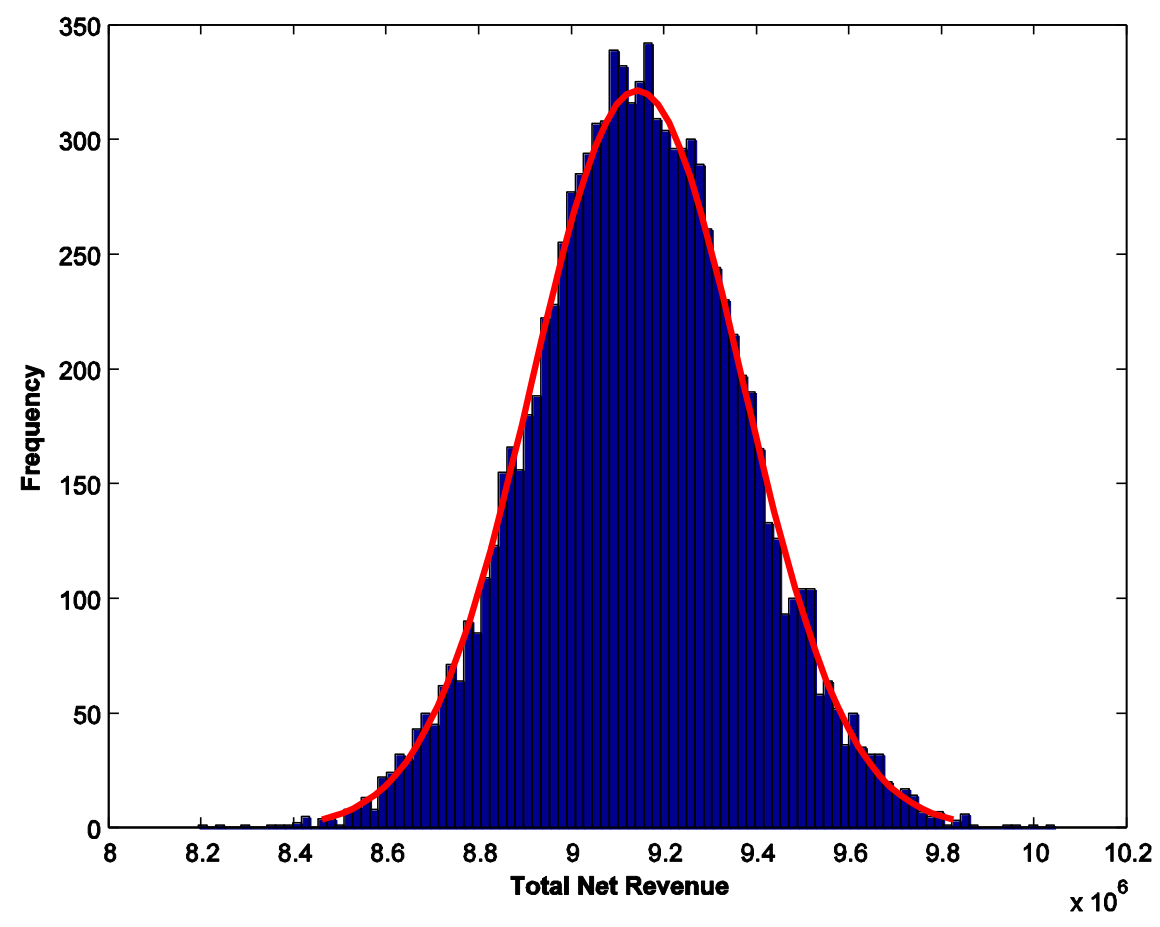

Figure 3 
Note that the sample standard deviation for the simulation was $\$ 227,400$. This is greater than the standard deviation in the unconstrained case indicating that the imposition of the quality constraint reduced expected revenues to the college and increases the dispersion. The shadow price of the quality constraint was a whopping $\$ 49.633 \mathrm{MM}$. At the margin (and the optimal solution), a .01 quality increase would decrease 'profit' by approximately $\$ 496,000$.

The simulated distribution for student quality under this scenario is depicted in Figure 4 .

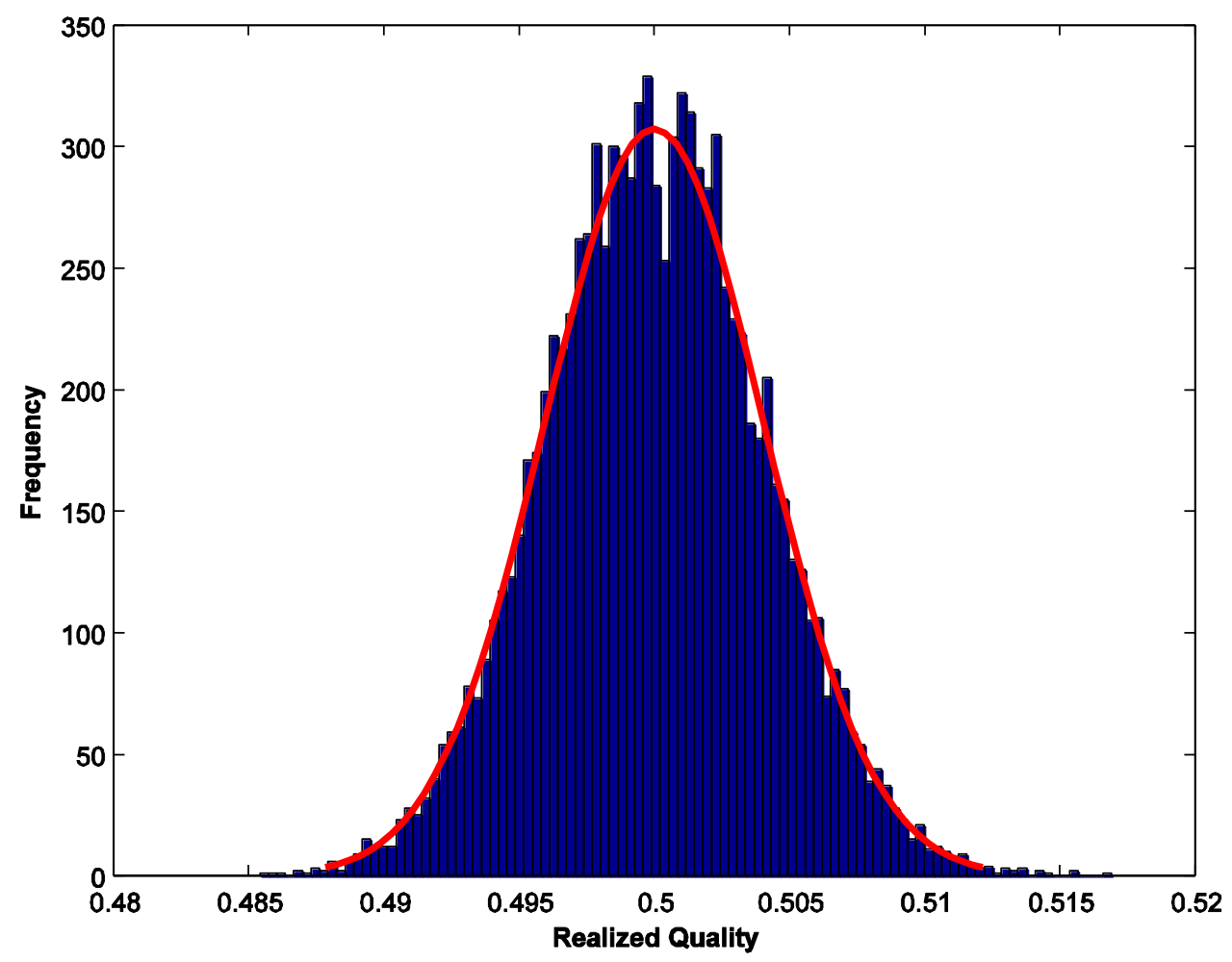

Figure 4

The sample standard deviation is .0041 which is higher than the unconstrained case. Again, the smooth curve denotes the comparable normal distribution.

\subsection{An Expected Capacity Constraint}

In our previous example where quality was unconstrained, we saw that the optimal tuition for the lower quality group was $\$ 5000$ and the expected enrollment was 2500 students. The optimal tuition for the higher quality group was $\$ 3000$ and the expected enrollment was 600 students. If expected enrollment was constrained to 2500 students (with no expected quality requirement), the optimal solution would be to charge tuitions of $T_{L}=\$ 5857$ and $\mathrm{T}_{\mathrm{H}}=\$ 3857$. Those levels would result in total expected tuition of $\$ 13.78 \mathrm{MM}$ and an average quality of .336 . The La Grange multiplier is $\$ 1714$, indicating that if the cost of an additional unit of capacity is less than $\$ 1714$ it should be added. Note that in this example, the capacity constraint reduces the average quality of the class. Somewhat surprisingly, the capacity constraint increases both low and high quality tuition by the same dollar amount.

While we have not explored them here, there are several possible alternative approaches to the capacity constraint formulation in this model. Based upon our approach, the enrollment distribution could easily be simulated. Different levels of expected enrollment could then be used to sort out the one which leads to a desirable 
probability of neither overutilizing nor underutilizing capacity. Alternatively, a penalty could be imposed in the simulation whenever enrollment exceeded capacity and the optimum could be directly simulated.

\subsection{Some Comparative Statics}

Some additional results associated with this sections two quality class case are discussed below. The first set of comparative statics deals with the responses of tuitions, profits, and enrollments as the depth of the high quality pool is varied. The results are based upon constraining expected quality to .5. Figure 5 displays the results of varying the pool depth for the high quality students between 1000 and 5000 (keeping the number of low quality students fixed at 5000, and all other parameters remaining the same). The figures indicate that $\mathrm{T}^{*}{ }_{\mathrm{L}}$ decreases as the pool depth for high quality students increases. This is a sensible result given that the model indicates these students are charged a premium as a result of the quality constraint. It is also the case that tuition decreases towards the $\$ 5000$ profit maximizing level but does not reach it. Tuition charged to the high quality class increases with the depth of the pool and moves towards the $\$ 2000$ optimum. The bottom panes in Figure 5 display the levels of expected enrollment and tuition profits based on the depth of the high quality pool.
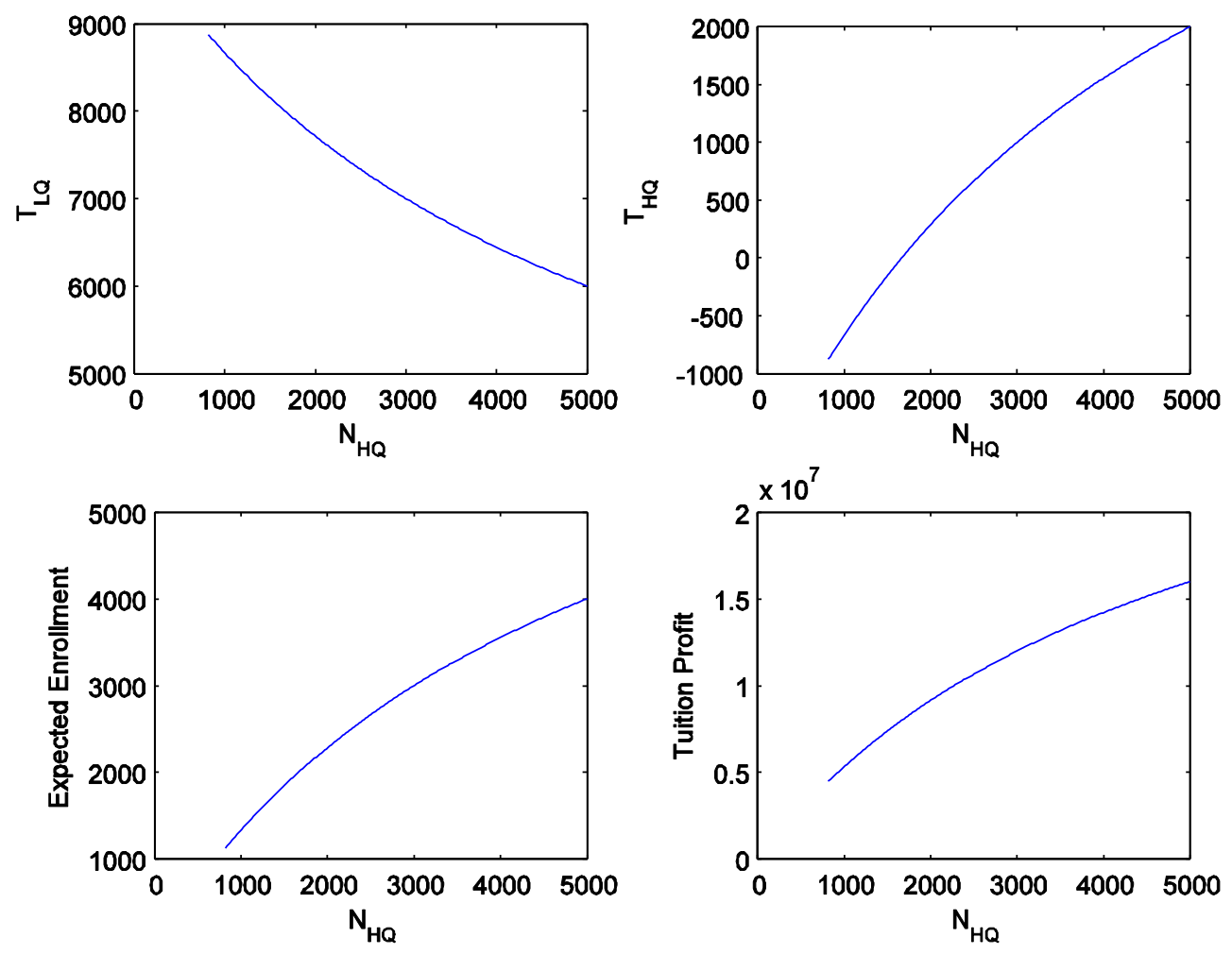

Figure 5

We perform a similar experiment for variations in desired quality in Figure 6. The top two diagrams depict the impact of changing the desired quality level from .25 to .75 on tuition charged to the low quality pool and the high quality pool. Both tuition levels start out flat at $\$ 5000$ and $\$ 3000$ respectively, because the unconstrained solution produces an expected quality level of approximately .35. Note that the school (monotonically) increases the tuition level to the low quality students to discourage their enrollment as desired quality is increased. This tuition charge is not monotonic for the high quality students however. At first, tuition is lowered and then starts to increase (at about $\bar{\alpha}=.5$ ). Associated with this behavior are shrinking expected enrollment and expected profit. 

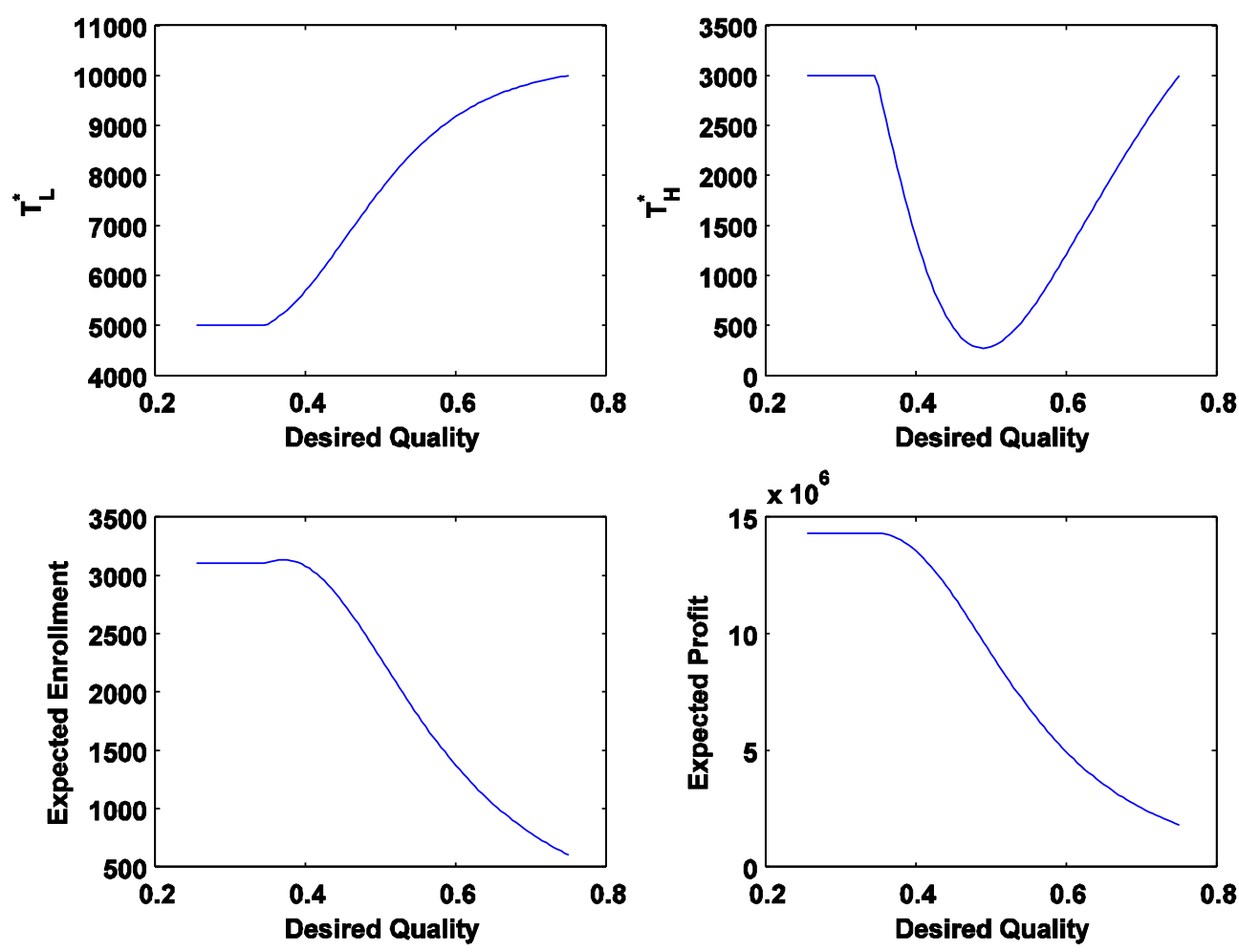

Figure 6

\subsection{Summary}

This section has explored the use of a linear model to examine the nature of the uncertainties associate with tuition, quality, and capacity decisions. The motivation for this model is its simplicity, potential estimability, and the opportunity it provides in a simulated environment to explore the risk implications of the decisions. We have explored how the optimal solutions are affected by the pool depths and constraints. The output from these comparative statics could be used in formulating long term objectives and strategies for the institution.

\section{CONCLUSION}

This paper has explored the use of an expected tuition profit model for decision making by the college or university. We have not argued that this is the 'correct' objective for the institution rather, our argument has been that in the absence of an objective function which reflects appropriate risk preferences, expected profit maximization is a reasonable starting point (especially for institutions facing financial difficulty). We adopted this view because many constituencies share in the residual risks of the less well endowed colleges. These include faculty, current and future students, and society. It is well established in the theory of the firm under uncertainty that appropriate utility functions representing the required blending of constituent preferences cannot generally be established even when the exact claims (risk-sharing) structure is apparent. We note that no such claims structure is apparent for most colleges or universities.

At some point, risk aversion and risk preferences must enter the analysis. We suggest that our model offers a starting point to presidents and directors or trustees, who wish to examine the risk consequences of their decisions. We point out that markets exist and always matter. To ignore the risk dimensions for some or all of the constituencies will lead to long run alternative economic choices by those units. 
A final point which we have neither focused on, nor have an immediate solution for, has to do with the type of information or data available. It is often difficult to take information collected by admissions and financial aid departments and translate the information into model inputs. We view the problem as one of unfocused information gathering. A model must be chosen and information gathering should be directed towards data usable in the context of the model, not the other way around. An exception to this appears to be Ehrenberg (1984) where the university seems to have collected enough information for him to explore the elasticities in an unconstrained setting.

\section{NOTES}

${ }^{1}$ See page 272 .

${ }^{2}$ See page 203 .

${ }^{3}$ See page 569 .

${ }^{4}$ See page 576 .

${ }^{5}$ See page 583 .

${ }^{6}$ See footnote 3 on page 631 .

${ }^{7}$ See page 5.

${ }^{8}$ For a discussion and exploration of the first point, see Wilson (1968).

${ }^{10}$ This is a rationality assumption in that the student only cares about net cost and not its composition. There may be tax implications which affect long run after-tax cost to students and the college should investigate this. In the absence of any such effects, students do not suffer money illusion in the sense that higher tuition offset by higher scholarships do not make the college more desirable. Nor do we accept the argument that high tuition is a quality signal. The existence of such an equilibrium requires that significant penalties be imposed on institutions and managers that falsely signal (apart from mere failure). We do not (casually) observe these penalties. A more legitimate criticism would seem to us to be that charging a high tuition (which will be discounted more), in the absence of appropriate name recognition, might well affect the matriculant's search pattern and lead to immediate removal of the institution from the search list.

${ }^{11}$ That is, $\varepsilon_{p}=(\partial p / p) / \partial T /(T-v)$

${ }^{12}$ One can think of net tuition, $T_{i}$, as a stated overall tuition, $T$, less a discount $\left(\mathrm{d}_{\mathrm{i}}\right)$ for each class, $d_{\mathrm{i}} \geq 0$. As mentioned previously, the resulting solution is not unique. To get around this we assume that the discount increases monotonically with the index of the quality pool. Furthermore, the overall tuition level is determined such that the low quality pool's discount is zero (i.e. $T=T_{L}$ ). More generally, we would let $T \geq \operatorname{Max}\left\{T_{i}\right\}$, then $d_{\mathrm{i}}=T-T_{\mathrm{i}}$

${ }^{13}$ That is, the limit of the decrease in expected profit from increasing the average quality from $\bar{\alpha}$ to $\bar{\alpha}+\mathrm{d} \bar{\alpha}$.

${ }^{14}$ It appears that the sign of $\lambda$ and $\alpha$ are related. For simplicity, we will assume the $\alpha$ 's are drawn from $0 \leq \alpha \leq 1$. Furthermore, by the envelope theorem:

$$
\Delta \text { Net Tuition Profit }=-\lambda N^{*} \mathrm{~d} \bar{\alpha}
$$

so that $\lambda$ may be interpreted as the negative of the change in total profit per enrolled student per unit of average quality.

${ }^{15}$ It should be observed that smaller schools (in terms of the pool depth) would appear to face greater relative uncertainty. This is due to the fact that the expected tuition increases linearly with the depth of the pool while the standard deviation increase with the square root of $N$. 
${ }^{16}$ Assuming independence, the standard deviation should be $\$ 187,163$.

${ }^{17}$ i.e. exactly to 4 decimal places.

${ }^{18}$ That is, the limit of the decrease in expected profit associated with increasing the average quality from $\bar{\alpha}$ to $\bar{\alpha}+\mathrm{d} \bar{\alpha}$.

${ }^{19}$ If $\mathrm{b}$ is changed to .0003 in this example, the number of enrolled students actually increases in the constrained case from 3100 to 3273 . Thus, the imposition of the constraint may increase space demand.

\section{AUTHOR INFORMATION}

Donald I. Bosshardt, Ph.D., is Associate Professor of Economics \& Finance at Canisius College. He received his doctorate in Finance from the University of Wisconsin at Madison. His research interests include corporate finance and portfolio management.

Larry Lichtenstein, Ph.D., is Associate Professor of Economics \& Finance at Canisius College. He received his doctorate in Economics from the State University of New York at Binghamton. His research interests include microeconomics and the housing market.

Mark P. Zaporowski, Ph.D., is Professor of Economics \& Finance at Canisius College. He received his doctorate in Economics from the State University of New York at Albany. His research interests include the behavior of interest rates, macroeconomics and applied econometrics.

\section{REFERENCES}

1. Bryan, Glenn A. and Thomas W. Whipple, "Tuition Elasticity of the Demand for Higher Education among Current Students," Journal of Higher Education, September/October 1995, Vol. 66, No. 5, pp. 560-574.

2. Ehrenberg, Ronald G. and Daniel R. Sherman, "Optimal Financial Aid Policies for a Selective University," Journal of Human Resources, 1984, Vol. 19, No. 2, pp. 202-230.

3. Jenny, Hans H., "Pricing and Optimum Size in a Non-Profit Institution: The University," Papers and Proceedings of Eightieth Annual Meeting of the American Economic Association, May 1968, Vol. 58, No. 2 pp. 270-283.

4. Martin, Robert E., "Tuition Discounting: Theory and Evidence,” Economics of Education Review, April 2002, Vol. 21, Issue 2, pp. 125-136.

5. Martin, Robert E., "Tuition Discounting Without Tears,” Economics of Education Review, April 2004, Vol. 23, Issue 2, pp. 177-189.

6. Rothschild, Michael and Joseph Stiglitz. "Equilibrium in Competitive Insurance Markets: An Essay on the Economics of Imperfect Information,” Quarterly Journal of Economics, November 1976, Vol. 90, No. 4, pp. 629-649.

7. $\quad$ Rothschild, Michael and Lawrence J. White. "The Analytics of Pricing in Higher Education and Other Services in Which Customers are Inputs," Journal of Political Economy, June 1995, Vol. 103, pp. 573-586.

8. Wilson, R., “The Theory of Sindicates,” Econometrica, 1968, Vol. 36, pp. 119-132. 


\section{NOTES}

